\title{
Ancho de distribución plaquetaria como predictor de preeclampsia severa
}

\author{
Platelet distribution width as a marker for the development \\ of severe preeclampsia
}

\author{
Carlos Andrés Trejos Ramírez MD ${ }^{1}$ Jorge Augusto Rodríguez Ortíz MD², Mortimer Arreaza Graterol MD, MSc ${ }^{3}$, \\ Fabiana Ramírez Pescador MD ${ }^{4}$ \\ ${ }^{1}$ Residente de cuarto año, Ginecología y Obstetricia. Universidad de la Sabana (Chía, Cundinamarca, Colombia). \\ Dirección electrónica: carlostrra@unisabana.edu.co \\ ${ }^{2}$ Ginecoobstetra, especialista en Epidemiología Clínica, Unidad de Medicina Maternofetal. Hospital Simón Bolívar (Bogotá D.C., Colombia). \\ ${ }^{3}$ Ginecoobstetra, especialista en Medicina Materno-Fetal, Unidad de Medicina Maternofetal. Hospital Simón Bolívar (Bogotá D.C., Colombia). \\ ${ }^{4}$ Médico, Universidad Santiago de Cali
}

\section{Resumen}

Introducción: La fisiopatología de la preeclampsia no está dilucidada por completo, diferentes índices plaquetarios dentro de los que se incluye el ancho de distribución plaquetaria (ADP) podrían ser utilizado para predecir la severidad en esta condición.

Objetivo: Analizar el comportamiento del ADP en el desarrollo de severidad en preeclampsia en mujeres atendidas en la unidad de medicina materno-fetal del Hospital Simón Bolívar de Bogotá, Colombia.

Materiales y métodos: Estudio de corte trasversal analítico en 105 pacientes con trastorno hipertensivo asociado al embarazo, preeclampsia y preeclampsia severa. Se analizó el comportamiento del ADP en una población de mujeres hipertensas con preeclampsia mediante una curva de análisis "Receiver Operating Characteristic" (ROC) para estimar la sensibilidad, tasa de falsos positivos, razón de probabilidad positiva y negativa de la prueba como marcador de desarrollo de severidad

Resultados: El ADP tuvo un ascenso significativo mayor en pacientes donde sus progresiones de enfermedad, desarrollaron características de severidad. En la curva ROC el área bajo la curva para del ADP como predictor de severidad en la preeclampsia fue de 0.68

Conclusión: El ADP es un índice plaquetario que aumentó significativamente en las mujeres con preeclampsia con características de severidad. El ADP podría ser un marcador para la predicción de severidad de la preeclampsia.

Palabras clave: pre-eclampsia, toxemia, trastornos de las plaquetas sanguíneas

\section{Abstract}

Introduction: The pathophysiology of preeclampsia is not completely elucidated. Different platelet indices, including the platelet distribution width (PDW), could be used to predict the severity of this condition.

Objective: To analyze the behavior of PDW in the severity development of preeclampsia in women treated in the maternal-fetal medicine unit of Hospital Simón Bolívar in Bogotá, Colombia.

Materials andmethods: This is an analytical cross-sectional study in 105 patients with hypertensive disorder associated with pregnancy, preeclampsia and severe preeclampsia. We analyzed the behavior of PDW in a population of hypertensive women with preeclampsia using a curve of analysis " Receiver Operating Characteristic " (ROC) to estimate the sensitivity, false positive rate, positive and negative likelihood ratio of the test as a marker of development of severity

Results: PDW had a higher significant increase in patients where their disease progression developed severity characteristics. In the ROC curve, the area under the curve for PDW as a predictor of severity in preeclampsia was 0.68 .

Conclusion: PDW is a platelet index that increased significantly in women with preeclampsia with severity characteristics. PDW could be a marker for the prediction of severity of preeclampsia.

Key words: pre-eclampsia, toxemia, blood platelets disorders. 


\section{Introducción}

La preeclampsia es trastorno grave de la presión arterial, se considera uno de los mayores problemas de salud durante el embarazo [1]. Puede complicar 3 al $8 \%$ de las gestaciones y causa un marcado incremento de la morbimortalidad materna; tan solo en Bogotá es la segunda causa de mortalidad materna (RR 1,99, IC 95\%: 1,78-2,22) [1,2,3].

Aunque la fisiopatología exacta de la preeclampsia es desconocida, algunos factores han sido atribuidos a ésta, dentro de los cuales se incluye la invasión trofoblástica alterada al compartimento vascular materno con la reducción subsecuente del flujo placentario [4,5]. La hipoperfusión placentaria es responsable del inicio de una disfunción endotelial materna sistémica e incremento de la permeabilidad vascular [6]. El sistema de la coagulación es activado por el contacto de las plaquetas con el endotelio lesionado llevando tanto a el incremento del consumo como a el aumento en la producción por parte de la médula ósea [7]. En la actualidad, diferentes índices son utilizados para medir la función plaquetaria, de entre los cuales, el ancho de distribución plaquetaria (ADP) que cuantifica la distribución del tamaño de las mismas [8]. Esta condición puede estar comprometida gracias a las alteraciones en red vascular placentaria. $(4,5,8)$

Cuando la mujer embarazada con un trastorno hipertensivo tiene un recuento plaquetario normal los parámetros relacionados a las plaquetas no son analizados secuencialmente hasta que presenta cambios significativos. Sin embargo, estudios han demostrado que la activación plaquetaria no controlada y la agregación de las mismas pueden darse en la preeclampsia con trombocitopenia y sin trombocitopenia [9]. La utilidad del ADP en la predicción de preeclampsia ha sido estudiado previamente [9-13]. En nuestro medio, autores como Cifuentes y cols han encontrado variaciones en sus resultados, dependiendo del trimestre de la gestación en donde este índice sea aplicado [10].

Éste estudio pretende analizar el comportamiento del ADP en mujeres atendidas por trastornos hipertensivos asociados al embarazo clasificadas como preeclámpticas de acuerdo al grado de proteinuria en 24 horas y al desarrollo o no de características de severidad en la unidad de medicina maternofetal del Hospital Simón Bolívar de Bogotá, Colombia.

Objetivo: Analizar el comportamiento del ADP en el desarrollo de severidad en preeclampsia en mujeres atendidas en la unidad de medicina materno-fetal del Hospital Simón Bolívar de Bogotá, Colombia.

\section{Materiales y métodos}

Diseño: Estudio de corte trasversal analítico.

Participantes: Para el estudio se tamizaron un total de 890 pacientes tomados de la base de datos del departamento de ginecoobstetricia del Hospital Simón Bolívar, institución de tercer nivel de atención de la Red Salud Norte del distrito en Bogotá, Colombia (desde noviembre de 2009 a noviembre de 2016). Las pacientes ingresaron al servicio por trastorno hipertensivo asociado al embarazo para estudio adicional. Se consideró la clasificación de cada paciente ingresada según las últimas guías del colegio americano de Obstetras y Ginecólogos (siglas en inglés ACOG) [14].

Finalmente se incluyeron en el estudio todas las pacientes con diagnóstico de preeclampsia en una cohorte única para analizar el comportamiento del ADP.

Posteriormente se realizó un subanálisis por subgrupos, los cuales fueron definidos conforme a la severidad del cuadro, ajustando la población a este criterio. Se definió como grupo uno a aquellas pacientes con preeclampsia severa y grupo dos a aquellas mujeres sin características de severidad en el curso de su enfermedad.

Se analizaron para ambas cohortes parámetros como la edad (en años), semanas de gestación, antecedentes ginecoobstétricos, tensión arterial, proteinuria, tipo de hospitalización, diagnóstico al ingreso, tipo de enfermedad hipertensiva, vía del parto y el ancho de distribución plaquetaria.

Todos los hemogramas fueron de quinta generación (FIA8000 de Getein Biotech, Inc), el cual cuenta con parámetros relacionados a las plaquetas, entre ellos el ADP, utilizado para fines del presente estudio.

\section{Análisis estadístico:}

Se realizó mediante el software de análisis de datos STATA 12, con el cual caracterizó la población con un análisis univariado. Los resultados de las variables cualitativas se describieron mediante frecuencias absolutas y porcentajes; para las variables cuantitativas se reportaron medidas de tendencia central, las cuales contaron con una distribución normal por lo cual fueron reportadas como media y desviación estándar (DE). Se realizó un análisis "receiver operating characteristic" (o curva ROC) para estimar la sensibilidad, tasa de falsos positivos, razón de probabilidad positiva y negativa de la prueba como marcador de desarrollo de severidad de la preeclampsia en la población descrita (Figura 1) 


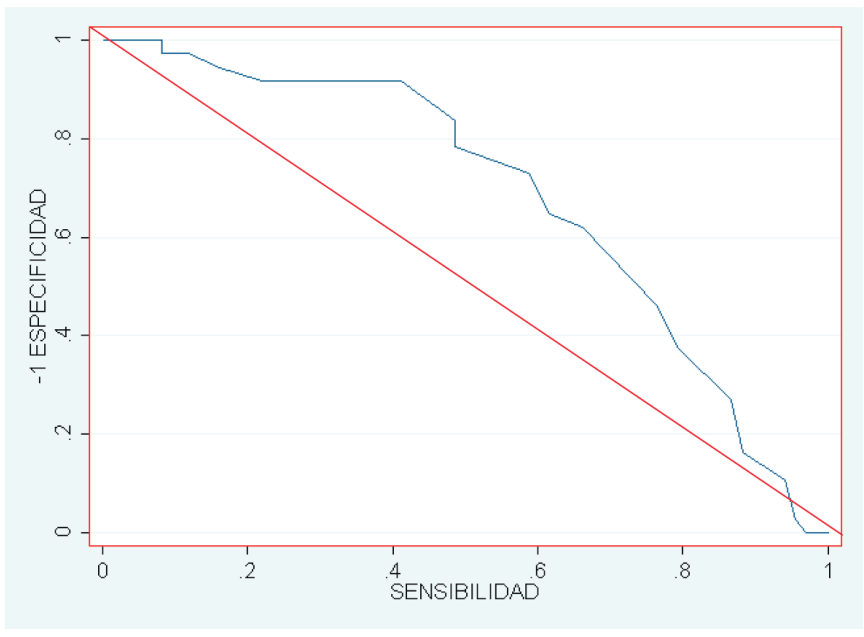

Figura 1. Análisis ROC de la población de mujeres intervenida

\section{Resultados}

La población reclutada fue de 105 mujeres quienes fueron ingresadas por urgencias de ginecoobstetricia al HSB. La edad promedio fue de 26 años (DE 6.81), El promedio de edad gestacional de la población reclutada fué de 35 4/7 semanas (DE 3.77), connotando una paciente con un aborto tardío sobre la semana $216 / 7$. El 43.81\% de las pacientes eran nulíparas contra el $56.19 \%$, quienes ya habían tenido más de un parto (tabla 1). Sólo un $22 \%$ de las gestantes tuvo un antecedente de trastorno hipertensivo previo (hipertensión crónica o trastorno hipertensivo asociado a embarazos anteriores). La cifra tensional promedio de la población en mención fué de 148/92 $\mathrm{mmHg}$, con un promedio de proteinuria en 24 horas de 454.9 $\mathrm{mg}$. El $5.71 \%$ de las pacientes requirió ingreso a la unidad de cuidado intensivo (UCI) por su patología en curso. Dentro del diagnóstico de trastorno hipertensivo asociado al embarazo, el $35.24 \%$ tuvo preeclampsia sin criterios de severidad, contra un $64.7 \%$ de pacientes quienes desarrollaron severidad. Entre éstos se subdistribuyeron según su compromiso orgánico. El $49.52 \%$ desarrolló criterios de severidad por compromiso de menos de 3 órganos, un $13.33 \%$ desarrolló síndrome HELLP (siglas del inglés, Hemolysis, Elevated Liver enzymes and Low platelets), una sola paciente terminó en eclampsia y una gestante desarrolló preeclampsia sobreagregada a una hipertensión crónica de base. La vía del parto fue en un $41.9 \%$ vaginal y en un $58.1 \%$ por cesárea. El peso promedio de los recién nacidos fué de 2429 gr (DE 0.687).

\begin{tabular}{|l|l|l|l|}
\hline Variable (n=105) & Media & Error estándar & CI 95\% \\
\hline Edad (años) & 26.1 & 0.664 & $24.7-27.4$ \\
\hline Edad Gestacional (semanas) & 35.4 & 0.368 & $34.7-36.2$ \\
\hline Proteinuria en 24 horas (mg) & 454.9 & 63.08 & $329.8-580$ \\
\hline Peso del RN (gr) & 2429.7 & 67.05 & $2296.8-2562$ \\
\hline
\end{tabular}

Tabla 1. Características de la población participante
El ADP tuvo un valor promedio de 16.6 (DE 0.611) (tabla 2). Para caracterizar el marcador plaquetario se realizó una curva ROC, con el fin de presentar la sensibilidad de la prueba en función de los falsos positivos obtenidos para la predicción de desarrollo de severidad de acuerdo a un punto de corte. De acuerdo a los cálculos realizados se encontró que el área bajo la curva fué de $68.4 \%$. El punto de corte con mayor sensibilidad y baja tasa de falsos positivos para la detección de severidad en la condición fue de 16.2 con $86.7 \%$ y $27 \%$ respectivamente. Éste punto seleccionado tuvo una razón de probabilidad positiva de 1.1890 y negativa de 0.4897 (ambos valores con peso estadístico adecuado).

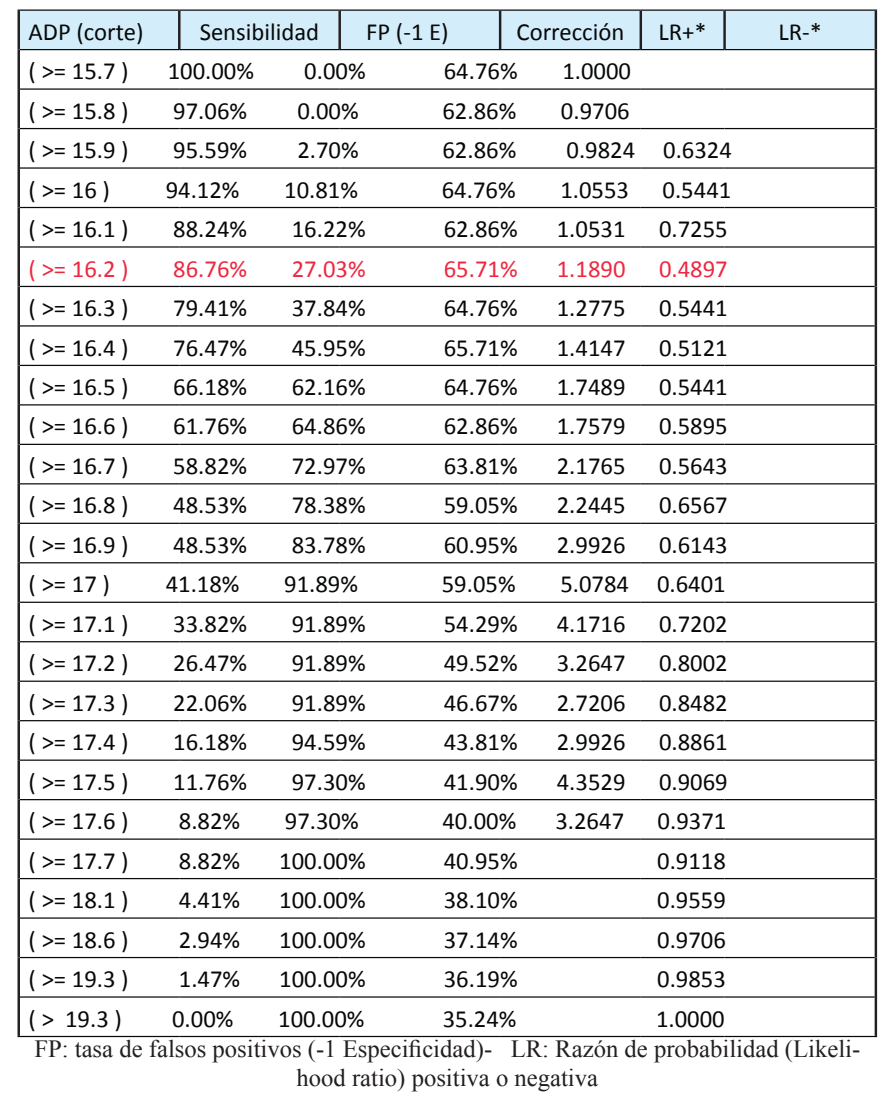

Tabla 2. Reporte de la sensibilidad del ADP de acuerdo al desarrollo de severidad por puntos de corte.

Dentro de las pacientes que requirieron UCI, que representa el $5.7 \%$ de las analizadas, el ADP siempre fue mayor que 16.2 en todos los casos. No se halló relación entre el grado de proteinuria en 24 horas y el nivel de ADP.

\section{Discusión}

Dado que la preeclampsia tiene una etiología desconocida, el diagnóstico se basa en la medición de la tensión arterial y la cuantificación de la excreción urinaria de proteínas [14]. Lo anterior indica que el clínico debe basarse fundamentalmente en la observación y ayudas paraclínicas. El desarrollo de severidad en el contexto de una preeclampsia eleva la mortalidad y cambia la conducta médica, por ejemplo, la 
decisión de finalizar la gestación de inmediato. Es por ello que los médicos pueden utilizar exámenes de laboratorio para predecir cuando la paciente está desarrollando características de severidad. En nuestro estudio el incremento del ADP fue mayor en las pacientes con preeclampsia con criterios de severidad comparado con las que no desarrollaron la misma. La activación plaquetaria en la preeclampsia se relaciona con la alteración del proceso de coagulación entre las plaquetas y las células endoteliales. Investigaciones previas han demostrado que la trombopoyetina plasmática incrementa en pacientes con preeclampsia como un marcador mayor de actividad plaquetaria [9-13]. Así mismo, el ADP como parámetro de activación plaquetaria, está incrementado en la preeclampsia. Éstos resultados sugieren un recambio activo de la producción plaquetaria por parte de la médula ósea, como resultado del consumo de plaquetas en sangre periférica, de acuerdo con estudios publicados por otros autores $[15,16,17]$. En consecuencia se debe tener en cuenta que el recuento plaquetario no es un indicador absoluto para la progresión de la preeclampsia y la alteración de la función y actividad plaquetaria debería ser evaluada.

Como la preeclampsia es un desorden multiorgánico, es difícil establecer marcadores de severidad para el desarrollo de la misma. Dundar y cols. sugirieron en un estudio que el volumen medio plaquetario podía ser utilizado como un marcador para la predicción del desarrollo de preeclampsia [18]. Sin embargo, no es clara la asociación entre índices plaquetarios y la predicción de severidad. En el presente análisis, el ADP en mujeres embarazadas con preeclampsia mostró un incremento significativo de regular calidad estadística en las pacientes que desarrollaron severidad, por lo cual se puede concluir que el ADP fué un marcador adecuado para predecir severidad en preeclampsia, por lo menos en aquella población intervenida en la institución.

Una limitación del estudio es la recolección retrospectiva de la información, lo cual no posibilitó la detección serial del ADP durante todo el período de gestación. No hay datos evaluados acerca del tiempo en el cual el ADP incrementa durante el embarazo [16].

\section{Conclusión}

El ADP fué un marcador útil para la predicción de severidad de la preeclampsia en la población estudiada de manera retrospectiva del centro descrito, que puede ser de valor en pacientes con plaquetas normales, antes de que la enfermedad progrese a severa o halla desarrollo de un síndrome HELLP. Se requiere de estudios prospectivos para validar la herramienta como instrumento medible para establecer asociación de la progresión a severidad de la preeclampsia, incluso en pacientes con recuento plaquetario normal.

\section{Agradecimientos.}

Al personal del área de archivo y del área de laboratorio clínico del Hospital Simón Bolívar de Bogotá D.C., quienes facilitaron datos de resultado del análisis de sangre de las pacientes para su posterior utilización en la estructuración del presente reporte.

\section{Conflictos De Interés.}

Ninguno declarado por parte de los autores.

\section{Referencias Bibliográficas.}

1. Adam GK, Bakheit KH, Adam I. Maternal and perinatal outcomes of eclampsia in Gadarif Hospital, Sudan. J Obs Gynaecol. 2009; 29(7):619-620.

2. Anderson UD, Olsson MG, Kristensen KH, Åkerström B, Hansson SR. Review: biochemical markers to predict preeclampsia. Placenta. 2012;33 Suppl:S42-S47.

3. Guia de trastornos hipertensivos del embarazo. Secretaria de salud distrital. Bogotá 2014.

4. Burton GJ, Woods AW, Jauniaux E, Kingdom JCP. Rheological and physiological consequences of conversion of the maternal spiral arteries for uteroplacental blood flow during human pregnancy. Placenta. 2009;30(6):473-482.

5. Burton GJ, Charnock-Jones DS, Jauniaux E. Regulation of vascular growth and function in the human placenta. Reproduction. 2009; 138(6):895902 .

6. Maynard SE, Karumanchi SA. Angiogenic factors and preeclampsia. Semin Nephrol. 2011;31(1):33-46.

7. Juan P, Stefano G, Antonella S, Albana C. Platelets in pregnancy. J Prenat Med. 2011;5(4):90-92.

8. Tzur T, Sheiner E. Is there an association between platelet count during the first trimester and preeclampsia or other obstetric complications later in pregnancy? Hypertens Pregnancy. 2013;32(1):74-82.

9. Hutt R, Ogunniyi SO, Sullivan MH, Elder MG. Increased platelet volume and aggregation precede the onset of preeclampsia. Obstet Gynecol 1994;83:1469.

10. Cifuentes-De la Portilla C, Chang-García MA . Variación del volumen plaquetario medio y el ancho de distribución de plaquetas como marcador clínico temprano de preeclampsia. Rev Colomb Obstet Ginecol . 2017; 68(2): 120-127.

11. Doğan K, Guraslan H, Senturk MB, Helvacioglu C, 
İdil S, Ekin M. Can platelet count and platelet indices predict the risk and the prognosis of preeclampsia? Hypertens Pregnancy. 2015;34(4):434-442

12. Han L, Liu X, Li H, et al. Blood coagulation parameters and platelet indices: changes in normal and preeclamptic pregnancies and predictive values for preeclampsia. PLoS One. 2014;9(12):e114488.

13. Karateke Atilla, Kurt Raziye Keskin, Baloğlu Ali. Relation of platelet distribution width (PDW) and platelet crit (PCT) to preeclampsia. Ginekol Pol. 2015;86(5):372-375.

14. American College of Obstetricians and Gynecologists, Task Force on Hypertension in Pregnancy. Hypertension in pregnancy. Report of the American College of Obstetricians and Gynecologists' Task Force on Hypertension in Pregnancy. Obstet Gynecol $2013 ; 122: 1122$.

15. Howarth S, Marshall LR, Barr AL, Evans S, Pontre $\mathrm{M}$, Ryan N. Platelet indices during normal pregnancy and pre-eclampsia. Br J Biomed Sci 1999;56:20-2.

16. Piazze J, Gioia S, Maranghi L, Anceschi M. Mean platelet and red blood cell volume measurements to estimate the severity of hypertension in pregnancy. $\mathbf{J}$ Perinat Med 2006;34:246-7.

17. Freitas LG, Alpoim PN, Komatsuzaki F, Carvalho MD, Dusse LM. Preeclampsia: are platelet count and indices useful for its prognostic. Hematology 2013;18:360-4.

18. Dundar O, Yoruk P, Tutuncu L, et al. Longitudinal study of platelet size changes in gestation and predictive power of elevated MPV in development of preeclampsia. Prenat Diagn 2008;28:1052-6. 